denoted respectively by $R, s$, and $\partial p / \partial x$, those for that component of the primary flow pertinent to $\psi^{\prime}$, denoted by $R^{\prime}, s^{\prime}$, and $\partial p^{\prime} / \partial x^{\prime}$, are determined by

$$
m^{\prime} R^{\prime}=m R, \quad m^{\prime} s^{\prime}=m s, \quad \frac{\partial p^{\prime}}{\partial x^{\prime}} \csc \beta^{\prime}=\frac{\partial p}{\partial x} \csc \beta
$$

in which $\beta$ and $\beta^{\prime}$ are the angles of inclination of the boundary to the horizontal in the $x$ - and $x^{\prime}$-directions, respectively, so that $s=\tan \beta$ and $s^{\prime}=\tan \beta^{\prime}$. As can be easily seen, the cross flow (in the $z^{\prime}$-direction) for the case of $\psi^{\prime}$ makes no consequential contribution to either the equations of motion or the equation of continuity, and does not affect in any way the satisfaction of the boundary conditions. Hence, the primary flow is stable or unstable for a three-dimensional disturbance according as it is stable or unstable for a two-dimensional one at a lower Reynolds number, a milder slope, and a reduced pressure gradient: the laws of reduction for the three quantities being given by Eqs. (6).

The writer arrived at the foregoing conclusion by using Squire's approach. Whereas many details were obtained as by-products, the rather cumbersome calculations involved are unnecessary if only the principal conclusion is desired. The general approach used here was suggested by the reviewer of the original manuscript of this paper, who attributed it to Professor C. C. Lin of the Massachusetts Institute of Technology.

\title{
ON DIFFUSION IN AN EXTERNAL FIELD AND THE ADJOINT SOURCE PROBLEM*
}

\section{BY JULIAN KEILSON (Lincoln Laboratory, M.I.T.)}

Abstract. If diffusion in an external field is described by $\partial \rho / \partial t=D \nabla^{2} \rho-\rho / \tau-$ $\nabla \cdot(\mathbf{F}(\mathbf{r}) \rho)$, the function $\gamma\left(r_{0}\right)$ describing the probability that a particle at $r_{0}$ will reach a collector surface before decaying or being absorbed by other surfaces satisfies the equation $D \nabla^{2} \gamma-\gamma / \tau+\mathbf{F}(\mathbf{r}) \cdot \nabla \gamma=0$. This equation has no singularity to disturb any geometric symmetry available. Boundary conditions on $\gamma(r)$ at the collector surface and other influencing surfaces are derived and shown to be independent of the external field. The boundary conditions at the secondary surfaces are homogeneous. The collector surface boundary condition is inhomogeneous.

1. Introduction. If particles with lifetime $\tau$ diffuse in the presence of an external field $\mathbf{F}(r)$, changes in density $\rho(r, t)$ are described by the continuity equation ${ }^{1}$

$$
\frac{\partial \rho}{\partial t}=-\frac{\rho}{\tau}-\nabla \cdot \mathbf{j}
$$

Here $\mathbf{j}$, the current density, is given by

$$
\mathbf{j}=-D \nabla \rho+\mathbf{F}(\mathbf{r}) \rho
$$

*Received April 17, 1953; revised manuscript received Dec. 31, 1953. The research in this document was supported jointly by the Army, Navy, and Air Force under contract with the Massachusetts Institute of Technology.

1J. Keilson, On the diffusion of decaying particles in a radial electric field, Journ. Appl. Phys., 24, 13971400 (1953). 
so the equation of motion is

$$
\frac{\partial \rho}{\partial t}=D \nabla^{2} \rho-\frac{\rho}{\tau}-\nabla \cdot(\mathbf{F}(\mathbf{r}) \rho) .
$$

Suppose one is interested in the probability $\gamma\left(\mathbf{r}_{0}\right)$ that a particle at $\mathbf{r}_{0}$ will reach the collector surface $S$ before decaying or being absorbed by surface $S^{\prime}$ (Fig. 1).

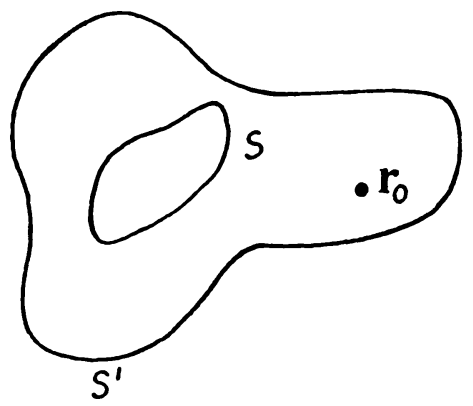

FIG. 1

To obtain $\gamma\left(r_{0}\right)$ one could solve the steady state diffusion equation for a source $I_{0}$ at $r_{0}$

$$
D \nabla^{2} \rho-\frac{\rho}{\tau}-\nabla \cdot(\mathbf{F}(\mathbf{r}) \rho)=-I_{0} \delta\left(\mathbf{r}-\mathbf{r}_{0}\right)
$$

with given boundary conditions at surfaces $S$ and $S^{\prime}$, and obtain $\gamma\left(r_{0}\right)$ as

$$
\gamma\left(r_{0}\right)=\frac{1}{I_{0}} \int_{0}\{-D \nabla \rho+\mathbf{F}(r) \rho\} \cdot d \sigma .
$$

It is often simpler to obtain $\gamma\left(r_{0}\right)$ as the solution of the homogeneous equation adjoint to $(4)$ :

$$
D \nabla^{2} \gamma-\frac{\gamma}{\tau}+\mathbf{F}(\mathbf{r}) \cdot \nabla \gamma=0 .
$$

The singularity at $\mathbf{r}_{0}$ no longer interferes with any geometric symmetry available, and the boundary conditions on $\gamma$, it will be shown, no longer contain the field $\mathbf{F}(\mathbf{r})$. The boundary conditions at the collector surface, however, are now inhomogeneous.

That $\gamma$ obeys Eq. (6) may be seen in the following way. Let $P\left(\mathbf{r}_{0} / \mathbf{r} ; t\right)$ be the solution of the time dependent diffusion equation (3), $t$ seconds after the particles were known to be at $\mathbf{r}_{0}$, i.e., that solution for which

$$
P\left(\mathbf{r}_{0} / \mathbf{r} ; 0\right)=\delta\left(\mathbf{r}-\mathbf{r}_{0}\right) .
$$

Continuity of probability demands that, for all $t$,

$$
\gamma\left(\mathbf{r}_{0}\right)=\int P\left(\mathbf{r}_{0} / \mathbf{r} ; t\right) \gamma(\mathbf{r}) d \mathbf{r}+\int_{0}^{t} d s \int_{s} j_{n}\left(\mathbf{r}_{s}, s\right) d \sigma .
$$

$j_{n}\left(\mathbf{r}_{s}, s\right)$ is the normal component of the current density at the point $\mathbf{r}_{s}$ on our collector surface $S$ at time s. At $t=0$, the collector current density hasn't had a chance to build up and $j_{n}\left(\mathrm{r}_{s}, 0\right)=0$. 
If then one differentiates Eq. (8) with respect to $t$ at $t=0$, it is seen that

$$
\int \frac{\partial \rho}{\partial t}\left(\mathbf{r}_{0} / \mathbf{r} ; 0\right) \gamma(\mathbf{r}) d \mathbf{r}=0,
$$

i.e.,

$$
\int\left\{D \nabla^{2} \delta\left(\mathbf{r}-\mathbf{r}_{0}\right)-\frac{\delta\left(\mathbf{r}-\mathbf{r}_{0}\right)}{\tau}-\nabla \cdot\left(\mathbf{F}(\mathbf{r}) \delta\left(\mathbf{r}-\mathbf{r}_{0}\right)\right)\right\} \gamma(\mathbf{r}) d \mathbf{r}=0 .
$$

When this is integrated by parts, Eq. (6) is obtained.

2. Boundary conditions. Suppose the boundary conditions on $\rho$ are

$$
j_{n}=-D \frac{\partial \rho}{\partial n}+F_{n}(\mathbf{r}) \rho=\alpha \rho \text { at } S,
$$

and

$$
-D \frac{\partial \rho}{\partial n}+F_{n}(\mathbf{r}) \rho=\alpha^{\prime} \rho \text { at } S^{\prime}
$$

The corresponding boundary conditions on $\gamma(\mathbf{r})$ at $S$, and $S^{\prime}$ are wanted. To find them one multiplies (3) by $\gamma(r)$, integrates over space and time, and obtains

$$
\int_{0}^{t} d s \int \gamma(\mathbf{r}) \frac{\partial P}{\partial t}\left(\mathbf{r}_{0} / \mathbf{r} ; s\right) d \mathbf{r}=\int_{0}^{t} d s \int \gamma(\mathbf{r})\left\{D \nabla^{2} P-\frac{P}{\tau}-\nabla \cdot(\mathbf{F}(\mathbf{r}) P)\right\} d \mathbf{r} .
$$

If one uses (6) and (7) and carries out integration by parts for both space and time one finds

$$
\begin{aligned}
\gamma\left(\mathbf{r}_{0}\right)=\int P\left(\mathbf{r}_{0} / \mathbf{r} ; t\right) \gamma(\mathbf{r}) d \mathbf{r} & +\int_{0}^{t} d s \int_{s}\left\{D P \frac{\partial \gamma}{\partial n}-D \gamma \frac{\partial P}{\partial n}+\gamma F_{n}\left(\mathbf{r}_{s}\right) P\right\} d \sigma \\
& +\int_{0}^{t} d s \int_{s^{\prime}}\left\{D P \frac{\partial \gamma}{\partial n}-D \gamma \frac{\partial P}{\partial n}+\gamma F_{n}\left(\mathbf{r}_{s}\right) P\right\} d \sigma
\end{aligned}
$$

But Eq. (8) tells us that

$$
\gamma\left(r_{0}\right)=\int P\left(\mathbf{r}_{0} / \mathbf{r} ; t\right) \gamma(\mathbf{r}) d \mathbf{r}+\int_{0}^{t} d s \int_{s}\left\{-D \frac{\partial P}{\partial n}+F_{n} P\right\} d \sigma .
$$

Comparing our last two equations it is seen that one must have for surface $S^{\prime}$ :

and hence

$$
D P \frac{\partial \gamma}{\partial n}-D \gamma \frac{\partial P}{\partial n}+\gamma F_{n} P=0,
$$

$$
D \frac{\partial \gamma}{\partial n}+\alpha^{\prime} \gamma=0 .
$$

This means that if, at $S^{\prime}$, one had perfect reflection $\left(\alpha^{\prime}=0\right)$, the condition on $\gamma$ there would be $\partial \gamma / \partial n=0$. If $S^{\prime}$ is perfectly absorbing $\left(\alpha^{\prime}=\infty\right)$, our condition on $\gamma$ is that $\gamma=0$. This is what one would expect since a particle near such a surface has little hope of reaching the collector surface.

At the collector surface, to agree with the continuity of probability equation one must have:

$$
-D \frac{\partial P}{\partial n}+F_{n} P=D P \frac{\partial \gamma}{\partial n}-D \gamma \frac{\partial P}{\partial n}+\gamma F_{n} P
$$


and hence

$$
\alpha=D \frac{\partial \gamma}{\partial n}+\alpha \gamma
$$

This condition states that at a perfectly absorbent collector surface $(\alpha=\infty), \gamma=1$. If the surface is perfectly reflecting $\partial \gamma / \partial n=0$, which is consistent with the more natural condition $\gamma=0$.

A typical application of the formalism above would be to the diffusion of decaying particles in the presence of a radial electric field. In the earlier paper ${ }^{1}$ the formalism is applied to the very simple case of a perfectly absorbing sphere about the center of symmetry.

I would like to thank Dr. S. F. Neustadter for his interest and encouragement.

\section{SOME PROPERTIES OF OPTIMAL LINEAR FILTERS ${ }^{1}$}

\section{Br HERBERT A. SIMON \\ (Carnegie Institute of Technology and Cowles Commission for Research in Economics)}

The purpose of this note is to discuss two considerations that arise in designing a filter that is optimal in the sense of minimizing the integral over time of some norm, under the further condition that we do not wish to employ information about the statistical structure of the signal. This is the "classical" problem of filter design prior to Wiener's work on stationary time series, although, in general, design was and is guided by figures of merit rather than by an explicit minimization procedure. It is probable that the results set forth here are well known, at least intuitively, to designers of servomechanisms, but I have not seen them set forth.

Since the norm to be miniminized is an integral over time, the problem may be viewed as one in the calculus of variations. Suppose that the norm, $F$, is:

$$
F=\int_{t_{0}}^{t_{2}} \phi\left\{y(t), y_{(1)}(t), \cdots, y_{(n)}(t)\right\} d t,
$$

where the integrand, $\phi$, is a polynomial in some variable, $y(t)$, and its first $n$ derivatives, $y_{(k)}(t)=d^{k} / d t^{k} y(t),(k=1, \cdots, n)$; so that we may write:

$$
\phi(t)=C+\sum_{i=0}^{n} b_{i} y_{(i)}+\sum_{i=0}^{n} \sum_{j=0}^{n} a_{i j} y_{(i)} y_{(i)}+\psi(t)
$$

where $\psi(t)$ is a polynomial composed of terms of third and higher degree in $y(t)$ and its derivatives.

To obtain a necessary condition for a minimum, we form Euler's equation:

$$
\phi_{0}-\frac{d}{d t} \phi_{1}+\cdots+(-1)^{n} \frac{d^{n}}{d t^{n}} \phi_{n}=0,
$$

where $\phi_{k}=\partial \phi / \partial y_{(k)}$.

${ }^{1}$ Received April 28, 1954. The results presented in this paper were obtained in the course of research undertaken by the Cowles Commission for Research in Economics under contract Nonr-358(01), NR 047-006 with the office of Naval Research. I am indebted to my colleagues A. Charnes, C. C. Holt, and F. Modigliani with whom I have had numerous valuable discussions on the topic of this note. 